\title{
Ainhum presenting to the accident and emergency department
}

\author{
M. HUNT \& E. E. GLUCKSMAN \\ Accident and Emergency Department King's College Hospital, Denmark Hill, London
}

\section{INTRODUCTION}

Ainhum (dactylolysis spontanea) is a poorly understood disease of unknown aetiology characterized by spontaneous auto-amputation of the fifth toe. The world-wide incidence of this condition is rare.

\section{CASE REPORT}

A 67-year-old man presented to the accident and emergency (A\&E) department of King's College Hospital referred by his General Practitioner (GP). He had developed a painful 'sore' on the medial aspect of his left little toe over the preceding month but had pain in the toe intermittently for some years. At the time of presentation the toe was attached only by a piece of thick skin on the lateral side of the toe, good volume peripheral pulses were present in the foot and sensation was normal (Fig. 1). He had no significant past medical history.

Radiological examination of the foot revealed autolysis of the middle phalanx and head of the proximal phalanx of the little toe with preservation of the distal phalanx (Fig. 2). Appearances were characteristic of ainhum.

The toe was amputated using local anaesthetic and the patient was given a course of antibiotics. The toe was redressed on two occasions and healed without complications.

\section{DISCUSSION}

The word ainhum may originate from the Naga dialect of Burma meaning to saw

Correspondence: M. Hunt, Consultant, Accident and Emergency Department Basildon Hospital, Nether Mayne, Basildon, Essex, U.K. 


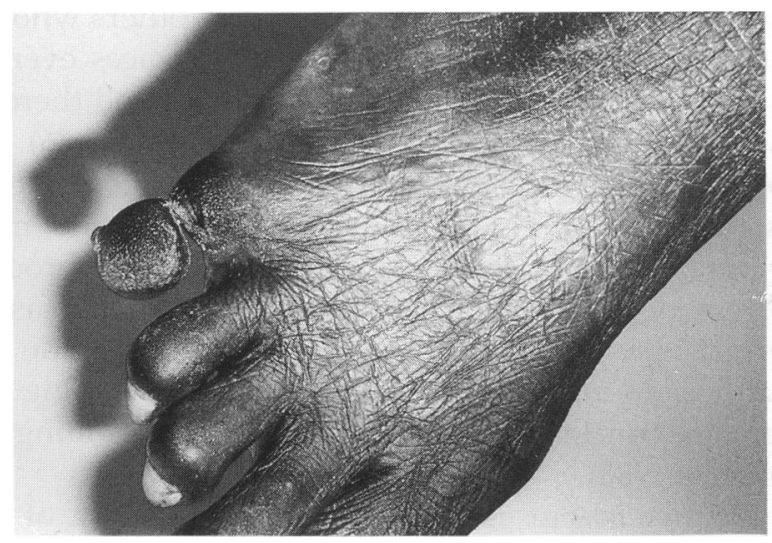

Fig. 1. Photograph showing toe attached on the lacerae side by a piece of thick skin.

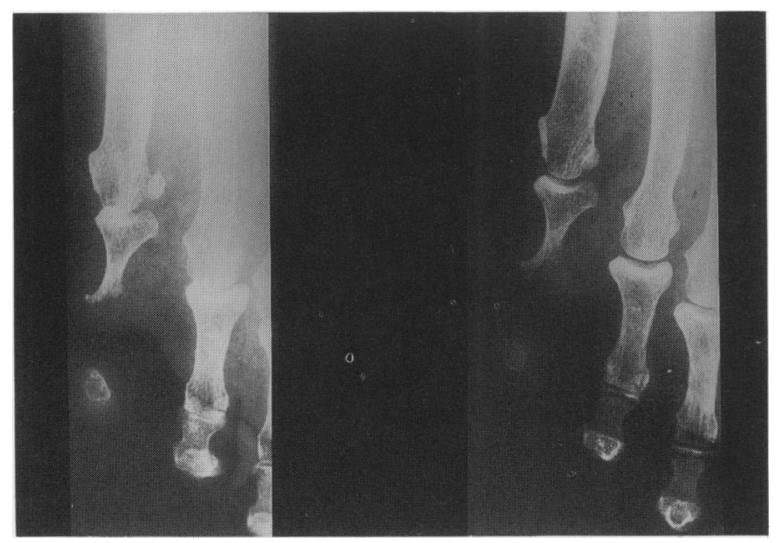

Fig. 2. Radiograph showing autolysis of middle phalanx and head of the proximal phalanx of the little toe.

or cut, or from Yoruba dialect as used in Brazil. The word is tendered most closely in English as 'ayan' meaning to saw or file. The medical terminology for this condition is dactylolysis spontanea.

Outside Africa ainhum is a very rare condition. The incidence varies between 0.2 and $2 \%$ in different African countries. It has been reported in the Middle and Far East, Central America and Caribbean Islands (Genakos et al., 1986; Kerhisnik et al., 1986) and rarely in the U.K. and U.S.A.

The aetiology of ainhum is unknown. It is said to affect males almost exclusively but review does not show a great difference in incidence between the sexes.

Racial and presumably genetic factors may predispose to the condition. Shoelessness in childhood and infection are said to be major factors. However it occurs 
in black people who have never gone barefoot and in others who have never worn boots or shoes. It has not been reported in non-black races even if they dispense with footwear. It is not due to infestation with parasites and there is no association with leprosy, yaws or sickle cell disease (Kerhisnik et al., 1986).

Radiological appearances are characteristic and diagnostic (Fetterman et al., 1986). Resorption or osteolysis of bone develops in the distal and middle phalanges, occurring in the outer layer of the cortex of the phalanx producing a tapering effect. Similar osteolytic lesion of the phalanges occur occasionally following electrical and thermal injury and exposure to polyvinyl chloride monomers. Osteolysis of bone, although uncommon, is recognized following trauma although the pathology remains unclear, early immobilization resulting in recalcification (Destoutet \& Murphy, 1983).

A number of authors favour ischaemia in the aetiology of osteolysis as the pathology resembles avascular necrosis. Other authors propose an autonomic mechanism producing secondary alteration in blood supply. Vascular occlusion produced by either thrombus or immune complex formation underlies osteolysis occuring in phalanges in cases of rheumatoid and psoriatic arthritis (Griffiths \& Glucksman, 1986).

The disease primarily affects the little toes either unilaterally or bilaterally (Genakos et al., 1986) and there are reports of it affecting the fourth and third toes, although these are very rare.

Initially a small corn or callus develops on the inner and plantar aspects of the little toe which progresses to a narrow band-like groove. Over the course of years the groove deepens and eventually encircles the toe until is falls off. Frequently fissure formation in the toe is accompanied by inflammatory changed from chronic sepsis resulting in swelling. Pain is reported to accompany the disease in $78 \%$ of cases and is intense. Clinically the usual differential diagnosis is that of atherosclerosis, but peripheral pulses are usually present and the vessels are normal on histological section.

Histologically, fibrosis occurs in the dermis beneath the groove with connective tissue fibres orientated longitudinally. Scanning electron micrographs suggest a fibrotic process thought to be analogous to that involved in keloid formation (Kerhisnik et al., 1986).

It is likely that the frequency of ainhum will increase in the U.K. as the number of immigrants rises. Ainhum has no distinguishing systemic manifestations or diagnostic laboratory tests, diagnosis being based on clinical and radiological features. Treatment of this condition is limited. In the early stages division of the fibrous band may delay evolution of the disease. Application of salicylic ointment may also delay progress, eventually however, surgical amputation may be required to relieve symptoms if auto-amputation does not occur.

Although painful this condition is of limited morbidity and may not present to medical practitioners, and when it does, it often goes unrecognized because of its rarity. 


\section{REFERENCES}

Destoutet J. M. \& Murphy W. A. (1983) Acquired acroosteolysis and acronecrosis. Arthritis and Rheumatism 26, $1150-1154$.

Genakos J. J., Cocores J. A. \& Terris A. (1986) Ainhum (dactylolysis spontanea). Report of a bilateral case and literature review. Journal of the American Podiatric Medical Association 76(12), 676-80.

Griffiths C. J. \& Glucksman E. E. (1986) Post-traumatic osteolysis of the clavicle: A case report. Archives of Energency Medicine 3, 129-132.

Fetterman L. E., Hardy R. \& Lehrer H. (1967) The clinicoroentenographic features of ainhum. Journal of Roentgenology, Radium and Therapeutic Nuclear Medicine 100(3), 12-22.

Kerhisnik W., O'Donnell E., Wenig J. A. \& McCarthy D. J. (1986) The surgical patholngy of ainhum (dactylolysis spontanea). Journal of Foot Surgery 25(2), 95-123. 